LAWRENCE LIVERMORE N A T IO N A L LABORATORY

\section{Source-Search Sensitivity of a Large-Area, Coded-Aperture, Gamma-Ray Imager}

K. P. Ziock, J. W. Collins, W. W. Craig, L. Fabris, R. C. Lanza, S. Gallagher, B. K. P. Horn, N. W. Madden, E. Smith, M. L. Woodring

November 10, 2004

IEEE Nuclear Science Symposium and Medical Imaging Conference Rome, Italy October 17, 2004 through October 23, 2004 
This document was prepared as an account of work sponsored by an agency of the United States Government. Neither the United States Government nor the University of California nor any of their employees, makes any warranty, express or implied, or assumes any legal liability or responsibility for the accuracy, completeness, or usefulness of any information, apparatus, product, or process disclosed, or represents that its use would not infringe privately owned rights. Reference herein to any specific commercial product, process, or service by trade name, trademark, manufacturer, or otherwise, does not necessarily constitute or imply its endorsement, recommendation, or favoring by the United States Government or the University of California. The views and opinions of authors expressed herein do not necessarily state or reflect those of the United States Government or the University of California, and shall not be used for advertising or product endorsement purposes. 


\title{
Source-Search Sensitivity of a Large-Area, Coded-Aperture, Gamma-Ray Imager
}

\author{
K. P. Ziock, J. W. Collins, W. W. Craig, L. Fabris, R. C. Lanza, S. Gallagher, \\ B. K. P. Horn, N. W. Madden, E. Smith, M. L. Woodring
}

\begin{abstract}
We have recently completed a large-area, codedaperture, gamma-ray imager for use in searching for radiation sources. The instrument was constructed to verify that weak point sources can be detected at considerable distances if one uses imaging to overcome fluctuations in the natural background. The instrument uses a rank-19, one-dimensional coded aperture to cast shadow patterns onto a $0.57 \mathrm{~m}^{2} \mathrm{NaI}(\mathrm{TI})$ detector composed of 57 individual cubes each $10 \mathrm{~cm}$ on a side. These are arranged in a 19 $x 3$ array. The mask is composed of four-centimeter thick, onemeter high, 10-cm wide lead blocks. The instrument is mounted in the back of a small truck from which images are obtained as one drives through a region. Results of first measurements obtained with the system are presented.
\end{abstract}

\section{INTRODUCTION}

$\mathrm{R}$ emote detection of small quantities of radioactive materials is of interest for a variety of applications. Although sufficient radiation can reach a large gamma-ray detector from a small source to make detection possible out to considerable distances, the naturally varying background makes such detections impossible unless it is known in advance. [1] Unfortunately, this is generally not the case.

In fact, because the background can vary by order of itself, and is unknown, the standard signal-to-noise ratio (SNR) expression:

$$
S N R=\frac{S}{\sqrt{B}} \propto \sqrt{A t}
$$

must be replaced by

$$
S N R=\frac{S}{B} \propto \frac{A t}{A t}=\text { Constant }
$$

Where $S(B)$ is the signal (background) count rate, in a detector of area, $A$, measuring for a dwell time, $t$.

A physical interpretation of this problem is that variations in the background at modest distances have the same shape in an omni-directional detector as a point source at that distance. Hence, a concrete building at $20 \mathrm{~m}$ will look like the point source one wishes to find.

Manuscript received October 29, 2004. This work was performed under the auspices of the U.S. Department of Energy by University of California, Lawrence Livermore National Laboratory under Contract W-7405-Eng-48.

K. Ziock, J. Collins, W. Craig, L. Fabris and N. Madden are with Lawrence Livermore National Laboratory, Livermore, CA 94550 USA (Corresponding author, K. Ziock, e-mail: ziock1@1lnl.gov).

R Lanza, B. Horn and S. Gallagher are with the Massachusetts Institute of Technology (e-mail: lanza@mit.edu,bkph@ai.mit.edu).

E. Smith and M. L. Woodring are with Pacific Northwest National Laboratory, Richland, WA, 99352

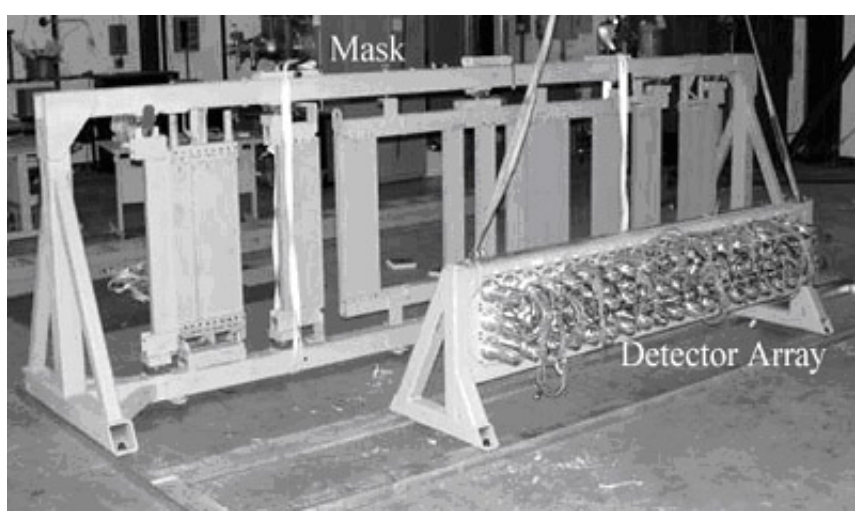

Fig. 1 The coded-aperture imager. The mask and detector are fixed to an aluminum frame that maintains the imager focal length and alignment. The system is mounted to look through the curb side of the vehicle.

To overcome this effect, we have developed an imaging instrument [2] that makes a map of the radiation field as it traverses a region. The pixel size of the map is sufficiently small at distances out to $100 \mathrm{~m}$ to allow one to distinguish point from distributed objects. The background in neighboring pixels thereby provides a measure of the background, allowing one to apply the classic SNR expression of equation 2, increasing sensitivity by an order of magnitude or more over an omnidirectional detector.

\section{INSTRUMENT}

\section{A. Mechanical design}

The imager (Fig. 1) is designed around an extant set of 57, $10 \mathrm{~cm} \times 10 \mathrm{~cm} \times 10 \mathrm{~cm} \mathrm{NaI}(\mathrm{Tl})$ detectors. These are arranged in a $3 \times 19$ array to sit behind a one-dimensional, base-19, uniformly-redundant array, coded-aperture mask [3]. The details of the design choices are provided in [2]. Briefly, the number of pixels in the imager is determined primarily by the length of time that an object is in the instrument's field of view, given its one meter focal length. This size is selected as a compromise between vehicle width and the imager resolution at $100 \mathrm{~m}(10$ $\mathrm{m})$. The imager provides only one-dimensional images - a design choice is based on its intended use in suburban or lighturban areas. Searches in areas with high-rise buildings beyond three stories are significantly complicated by the extra shielding from the concrete floors prevalent in their designs.

The mask comprises individual elements of linotype metal ( $85 \% \mathrm{~Pb}, 11 \% \mathrm{Sb}, 3 \% \mathrm{Sn}$, and $1 \%$ other materials) that are 4 $\mathrm{cm}$ thick by $10.8 \mathrm{~cm}$ wide by $100 \mathrm{~cm}$ high. This material was 


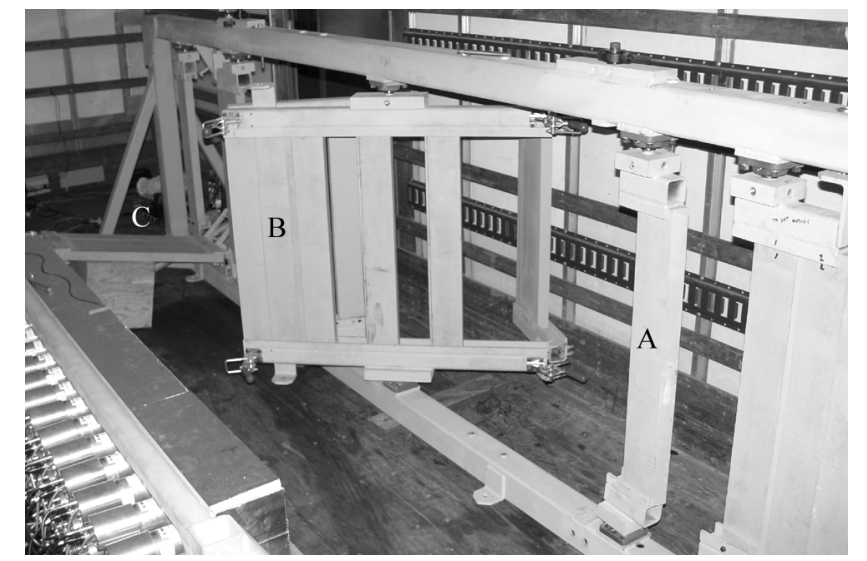

Fig. 2. The mask is converted to the anti-mask by rotating different parts about axis of anti-symmetry ( $\mathrm{A}$ and $\mathrm{B}-\mathrm{B}$ is hinged to reduce the swing space required) or by lowering a section out of the field of view (C).

selected for its lead-like stopping power with improved mechanical properties. The individual elements are supported in a steel frame that allows for rotation of sections of the mask around axis of anti-symmetry. Additional mask elements may be lowered into or out of the field of view of the detector (Fig. 2). These features allow us to exchange the mask for its inverse (anti-mask) in sequential passes past a source, providing an important means of noise control as explained further below.

The detectors are supported from a plate mounted in a steel frame similar to that used for the mask. The detectors are separated from the plate with a $0.5 \mathrm{~cm}$ thick sheet of elastomer to help protect them from mechanical and thermal shock. An additional $2.5 \mathrm{~cm}$ of Styrofoam is placed on the front, tops, sides and bottom of the detector array as thermal insulation.

Several pieces of aluminum channel connect the mask and detector frames to fix the one meter focal spacing and to restrict motion of one with respect to the other. The instrument relies on friction to keep it from moving within the truck. A safety strap ties the mask to the rear of the vehicle to protect the cab occupants in the event of a sudden stop. The instrument weighs $1585 \mathrm{~kg}$ with the $1225 \mathrm{~kg}$ mask comprising the bulk of this mass. The overall length of the mask is four meters.

\section{B. Electronics Design}

The low-power electronics (120 Watts, full system) runs off the vehicle power system. It is based on a modular concept with one circuit board per detector (Fig. 3). The card supplies both a fast channel for triggering and a slow channel for spectroscopy. Signals above a lower level threshold trigger the card which digitizes the output of the slow channel using a low power 16-bit digitizer (decimated to 12 bits to achieve nuclear quality.) The logic also sets a signal to alert the overall system that the card has data. Up to 64 of these cards can populate a motherboard that provides communications to a centrallylocated, digital-processing card.(Fig. 4).

A field-programmable gate array (FPGA) mounted on this board reads the $\mathrm{ADC}$ values and forms a 32 bit digital word by

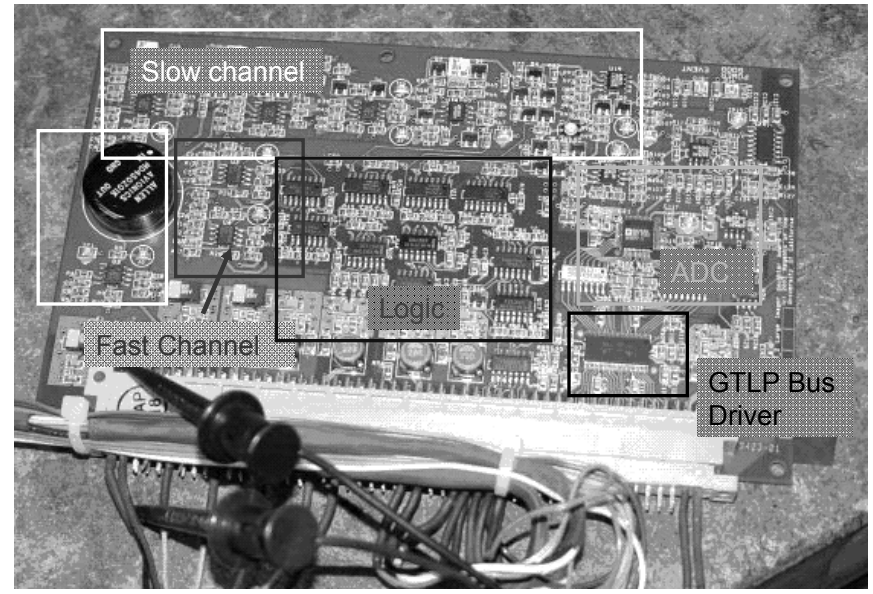

Fig. 3. Single channel PC board with different regions identified.

adding the card number and whether upper and lower discriminators (not used in this experiment) have fired. The FPGA sends the data to a commercial PCI card [4] for processing by the data collection computer. In addition to servicing the individual cards, the FPGA card also maintains a system clock. The value of this clock is inserted at one-millisecond intervals into the data stream. Finally the FPGA card accepts events from a commercial, Doppler-radar-based "fifth-wheel" that provides location information. [5] This unit generates a frequency of $100 \mathrm{~Hz}$ for each mile per hour of the vehicle. The FPGA board counts the pulses from this unit and provides two sequential 32-bit events (ID plus 16 bits of the count per event) every 10 counts. If the vehicle is not moving, the location count is provided every second).

\section{DATA HANDLING}

The data-collection computer is mounted with the other electronics in the back of the vehicle. A local area network is used to provide the vehicle passenger with control of the system via a laptop computer. The high level control and display software is written in Microsoft Visual Basic 6. [6] Data is collected from the National Instruments card using their NI DAQ function calls. As the data is collected, an event-mode file of the full data stream is recorded to disk for later analysis. Simultaneously, the data is parsed for image processing and display. On-line displays include a grayscale view of the $19 x$ 3 detector assembly, energy histograms of individual pixels or the entire detector array, a counts-per-meter histogram and the image of the track past the source.

The displays are populated from the incoming data using the following methods. First, the raw data is corrected for gain and offset based on stored calibration data from previous runs with ${ }^{137} \mathrm{Cs}$ and ${ }^{60} \mathrm{Co}$ sources. The linearized data is then checked against possible limits in both position and energy before it is added to an energy histogram maintained for each of the 57 detectors and a "short-term" detector. The later is a 19-element array that is used to generate the images as described below. 


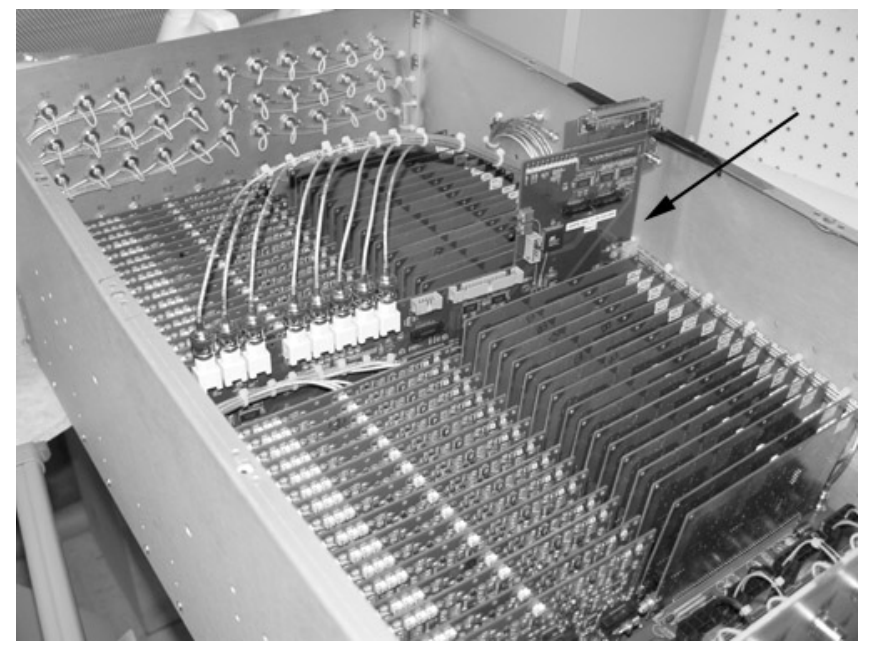

Fig. 4. Electronics assembly in a standard 19 inch rack-mount chassis. The FPGA board (arrow) services the 64 individual cards via the mother board.

\section{A. Image Generation}

As an image run proceeds, data is continuously summed to the short-term detector until a fifth-wheel event occurs. Upon such an event, the current location of the imager is checked against one of 11 possible pixel boundaries. These boundaries represent the edge of "world pixels" at different distances from the detector and are the key to understanding how an overall image such as that shown in Fig. 6 (top) is created. The simplest of these is used to generate the counts-versus-position histogram (Fig 6, bottom) and is explained first.

For the counts-versus-position histogram, the pixel boundaries occur every meter and the histogram simply keeps track of the number of counts that occur while the imager is within that one meter distance. Upon a fifth-wheel event, if the detector position does not exceed the pixel boundary, then the total counts from the short-term detector are summed into a register specific to this histogram. If the location exceeds the pixel boundary, then the counts already in the register are normalized to the expected dwell time in the pixel and placed in the appropriate pixel of the counts-per-meter histogram, the register is cleared, the time is recorded, the new data is used to initialize this register and the next pixel boundary is calculated.

Normalization to the dwell time is performed by taking the actual time spent in the pixel (current time minus the start time for the pixel) divided by the expected dwell time at the estimated velocity. The later number is estimated at the start of the run and is the velocity that the driver attempts to maintain during the scan. Note that this number is arbitrary and the same relative image results although the numeric values differ if one uses a different assumed velocity.

The procedure to construct the overall image is only slightly more complicated. In this case we have ten different worldpixel maps to keep track of - one for each 10-meter distance increment from the imager. These pixel maps have different boundaries since the size of the pixels increases as the distance from the imager increases. The choice of ten, 10-meter pixel maps is arbitrary; one could just as easily have selected 100 , one-meter pixel maps. However, the distance information is limited by the parallax as one passes a source and more pixels increase the processing required. No optimization of the number of distance pixels has been performed at this time.

Consider the world-pixel map centered at $55 \mathrm{~m}$ that is meant to include sources from 50 to $60 \mathrm{~m}$. For a $1 \mathrm{~m}$ focal length, the pixels are $5.94 \mathrm{~m}$ wide. The absolute edge of a pixel is selected in the code by assuming the pixels for all ranges line up at location "zero" halfway through the scan (again this is selected before the pass and is arbitrary.) During the scan, (see Fig. 5) when a fifth-wheel event occurs, the code checks to see if the edge of the current $5.94 \mathrm{~m}$ wide pixel has been reached. If not, then the data in the 19-element, short-term detector is summed to a 19-element, "distance-specific" detector array for this distance. If the edge of the pixel is exceeded, then the data in the distance-specific detector array is normalized to the dwell time and then used to form an image based on the coded aperture deconvolution algorithm. [7] This provides a 19-element image that is added to the correct 19 elements of the world map at the $55 \mathrm{~m}$ range. (The center of the image maps to the current location.) The distance-specific array is zeroed, and a new start time and pixel boundary are recorded for this source range. Finally, the grayscale histogram is updated and more data is processed.

\section{B. Mask/Anti-Mask data handling}

The imager can be run with the mask in either a "mask" or an "anti-mask" configuration. These different configurations are used to remove spurious background from the images as originally pointed out in [8]. Specifically, the coded aperture image process generates an image based on the variation in counts versus position across the face of the detector. These variations are supposed to be due to the shadow cast by the mask on the detector. However, variations are also possible due to radiation scattered off of nearby structures, radiation incident from other directions and even shielding of inside pixels by those on the edge of the detector array. A summation

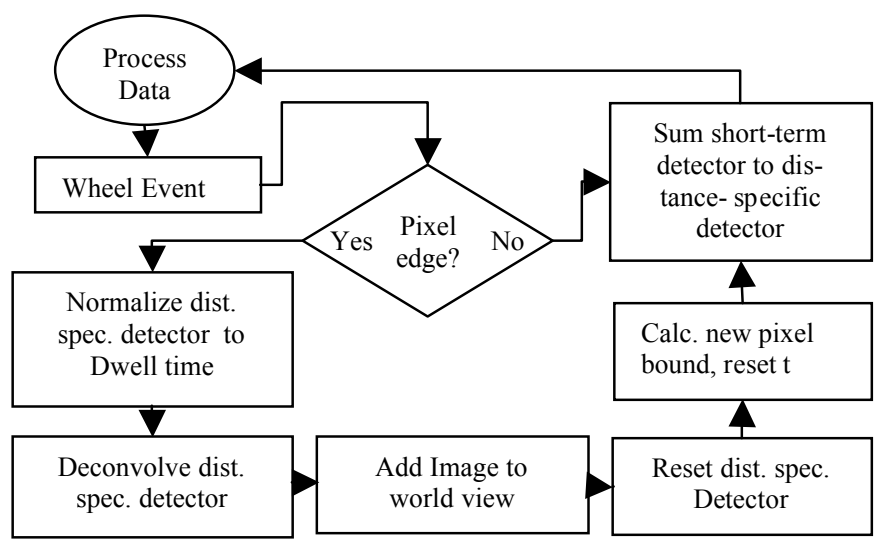

of equal time exposures with the mask and its inverse should show no variation of counts versus position. Any variations

Fig. 5. Flow diagram used to generate world image. 

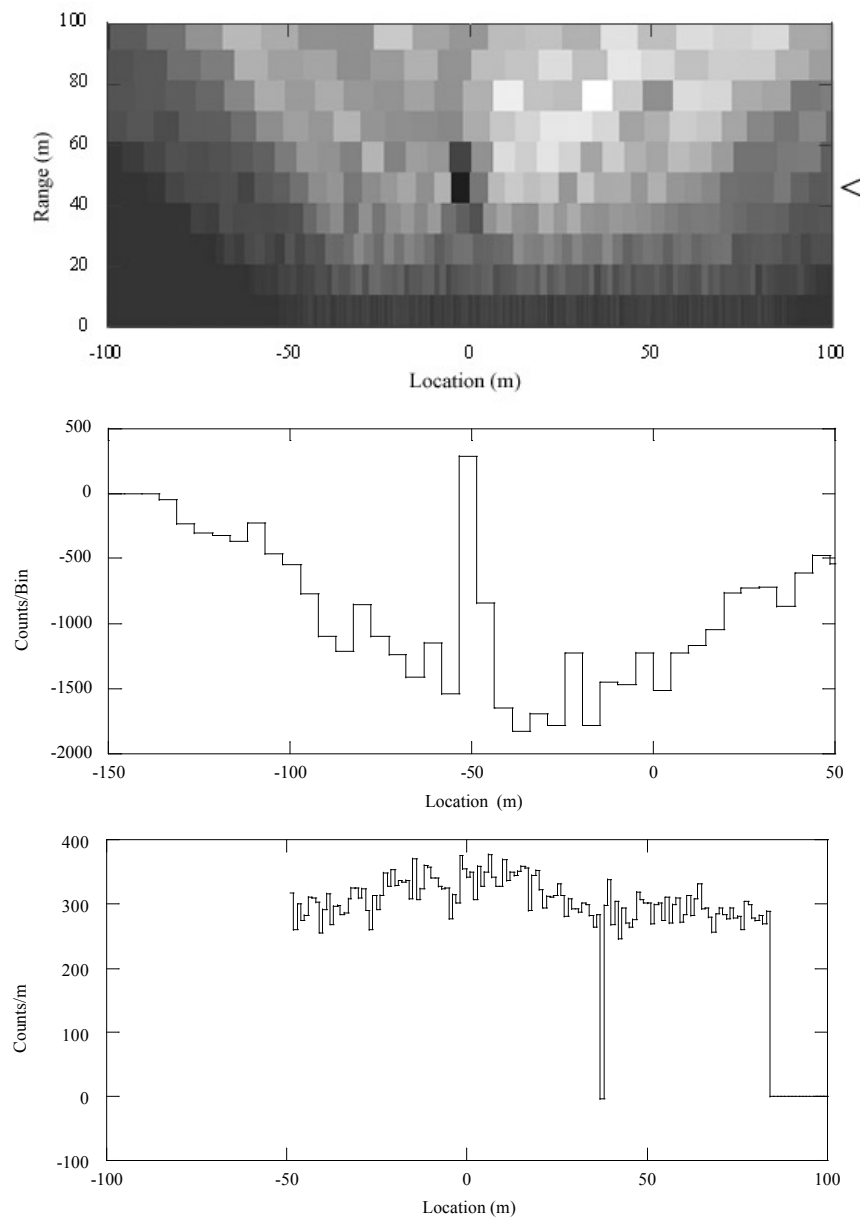

Fig. 6. Anti-mask track past $1 \mathrm{mCi}{ }^{137} \mathrm{Cs}$ source at $\sim 5 \mathrm{~km} / \mathrm{h}$, range $46 \mathrm{~m}$ (top). The middle plot shows the histogram of counts vs position at the distance denoted by "<" (45 m). The bottom plot is the counts-per-meter histogram. Note that this does not cover as wide a range as the imager data. The drop out at $\sim 45 \mathrm{~m}$ is an artifact.

that exist are extraneous and introduce artifacts in the image. The mask plus anti-mask data set can be subtracted from each of the separate runs before the image is generated to remove this noise. However, as shown in [9], this procedure is the same as adding mask-only data and subtracting anti-mask only data from the image. This is the approach taken in here, so that if the mask is in the anti-mask configuration, the data accumulated in the short-term detector is subtracted from the distancespecific detector. The image generation multiplies these values by plus or minus one so that both positive and negative counts can occur in an image. In general the mask (anti-mask) only image is greater (less) than zero due to the non-modulated background in the detector (see Fig. 6.)

\section{Error estimation}

To first order, the statistical error in any single pixel of a coded aperture image is given by the square root of the total number of counts in the entire detector. [10] This follows since the image deconvolution process is linear. In this work, this simple observation is complicated by the fact that multiple images, each normalized to their own dwell time, are added to generate the final world image at any given distance from the detector. Therefore the variance in any given pixel, $\delta I$ is:

$$
\left(\delta I_{n}\right)^{2}=\sum_{j} N_{j} / D_{j}^{2}
$$

where $N_{j}$ is the number of counts in the $j^{\text {th }}$ image and $D_{j}$ the appropriate dwell time for that image. To keep track of this, an additional summation is made whenever a pixel boundary is encountered and a world map of this sum is maintained to provide the variance at any given pixel of the final image.

\section{PERFORMANCE}

The completed imager was used to track past a one millicurie ${ }^{137} \mathrm{Cs}$ source at a number of different velocities and distances. In all runs, only data in the $662 \mathrm{keV}$ peak (590 - 730 $\mathrm{keV}$ ) is used. Distances were fixed by the local parking lot and road structure and the requirements that the source remain inside its "assigned" building. For the first measurements, the source is mounted against the inside of the near wall of the building and the vehicle tracked past the source at a distance of $46 \mathrm{~m}$ at the slowest velocity that could be maintained reliably $(\sim 5 \mathrm{~km} / \mathrm{h})$. The building itself is a tall corrugated metal-sided structure with apparent minimal wall thickness defined by the metal and insulation, both of unknown thickness.

The track past the source at $46 \mathrm{~m}$ with the imager in the antimask configuration is shown in Fig. 6. The top image is the complete grayscale image for the full set of distances on $10 \mathrm{~m}$ centers. The source is clearly visible in the image. The middle panel shows the count histogram of the $45 \mathrm{~m}$ distance, clearly revealing a significant detection ( \pm 162 counts, one sigma). The source is also visible as the broad "bump" in the counts-
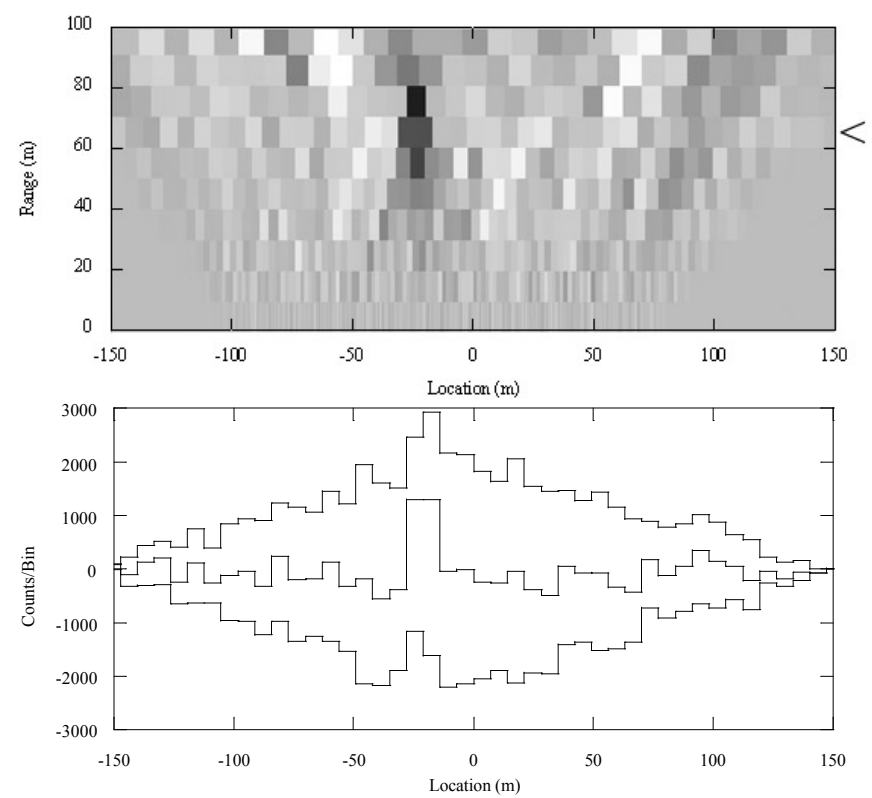

Fig. 7. Combined mask/anti-mask image of track past the source at $5 \mathrm{~km} / \mathrm{h}$ at a range of $66 \mathrm{~m}$ (top.) The bottom plot shows the histograms of the image at $65 \mathrm{~m}$ denoted by the "<" with the top curve the mask pass, the bottom curve the anti-mask pass and the middle curve the final image using both. 

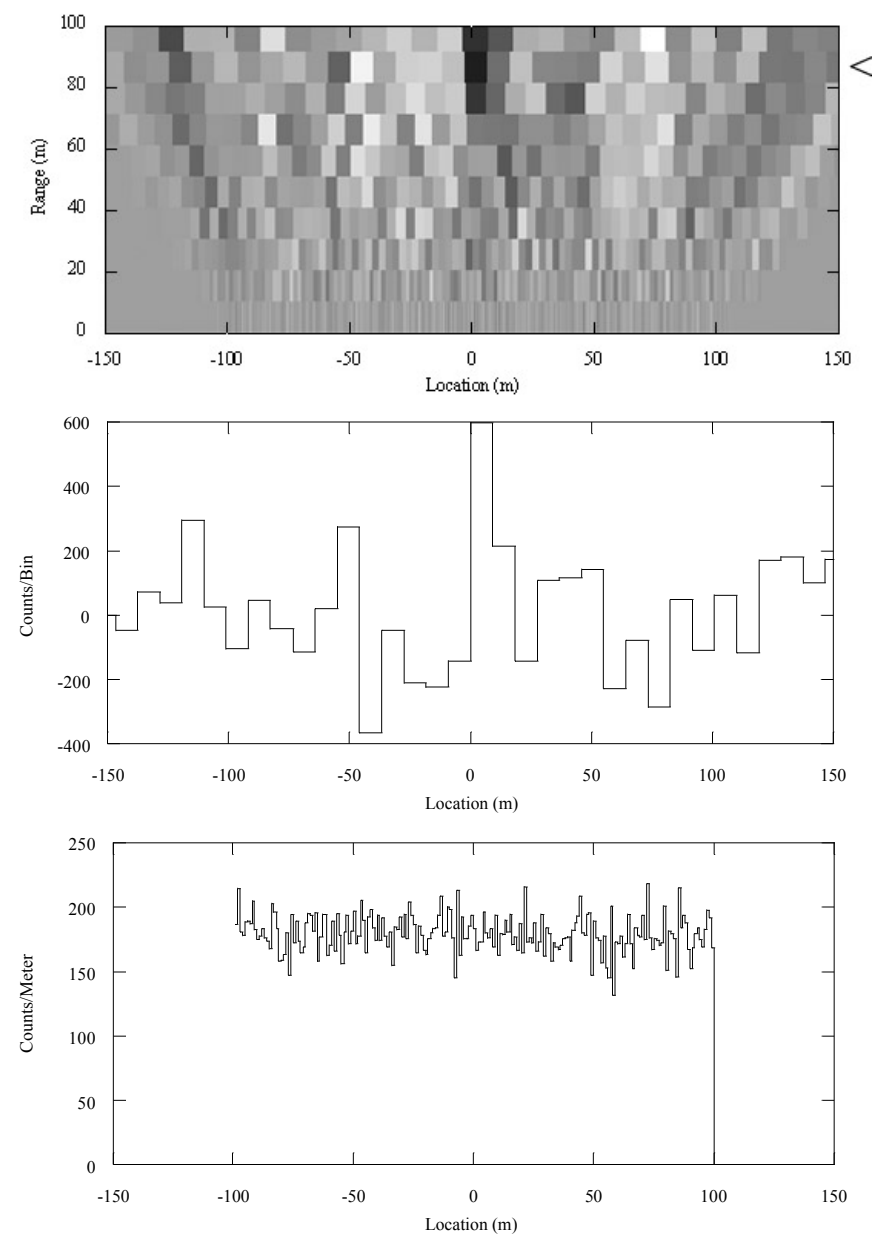

Fig. 8. Combined mask/anti-mask image of source at $16 \mathrm{~km} / \mathrm{h}$ at a range of $83 \mathrm{~m}$ (top). The graph in the middle is the histogram of the image at $85 \mathrm{~m}$ denoted by the "<". The peak is three sigma above zero counts. The histogram at the bottom is the counts per meter in the detector.

per-meter histogram shown at the bottom of the figure. However, the bump is small enough, that the assignment as a point source could not be made with certainty without imaging.

In Fig. 7 we present the results for a track past the source at a range of $66 \mathrm{~m}$, also traveling at $5 \mathrm{~km} / \mathrm{h}$. In the grayscale image at the top of the figure we show the combined mask/antimask data. This no longer suffers from the large offset in counts as observed in Fig. 6. The histogram at $65 \mathrm{~m}$ is plotted below the image for the mask pass, the anti-mask pass and for the combined data as shown in the image. The offsetting nature of the two passes is clearly evident.

Finally, we moved the source to the far side of the building to obtain a track past the source at $83 \mathrm{~m}$. This image (Fig. 8) was obtained traveling at $16 \mathrm{~km} / \mathrm{h}$. It also includes effects from all of the structural components of the near wall of the building. The counts histogram at $85 \mathrm{~m}$ is given in the middle panel of the figure. The peak represents a three-sigma departure from zero counts indicating that a detection has been made. For comparison, the counts-per-meter histogram is shown in the bottom panel. It is hard to imagine an argument that would allow one to conclude that the source is present based on that data alone.

\section{CONCLUSions}

It is clear that the imager allows one to determine the presence of the source utilized out to distances beyond $80 \mathrm{~m}$. However, at three sigma, the detection at the furthest range is positive only based on the a priori knowledge that the source is present. (A track without the source present shows a maximum peak at a different location with a two sigma excursion above zero counts.) It is clear that greater statistical significance is required for a true search where the number of false alarms would be too high if all three-sigma "events" were investigated.

A number of further measurements will be undertaken in the near future to better understand the properties of the background in the imager. These will be used to define an improved instrument. Improvements must include the mask/antimask information in a single pass and a detector pixel size that is optimized for the background found in these additional measurements.

\section{ACKNOWLEDGEMENTS}

The authors wish to thank the LLNL LDRD office for early support of this project and the Department of Homeland Security for current support. Mechanical assistance provided by Dennis Carr and Darrel Carter of LLNL is also gratefully acknowledged.

\section{REFERENCES}

[1] K.P. Ziock, W. Goldstein, "The lost source, varying backgrounds and why bigger may not be better," in Unattended Radiation Sensor Systems for Remote Applications, J. Trombka, D. Spears, P. Solomon, editors, AIP Conf. Proc. vol. 632, pp. 60-70, 2002.

[2] K.P. Ziock, W.W. Craig, L. Fabris, R.C. Lanza, S. Gallagher, B.K.P. Horn, N.W. Madden, "Large Area Imaging Detector for Long-Range, Passive Detection of Fissile Material," IEEE Trans. Nucl. Sci.Vol. 51, no. 5, pp. 2238-2244, 2004.

[3] S. Gottesman, E. Fenimore, "New family of binary arrays for coded aperture imaging," App. Opt., vol. 28, No. 20, pp. 4344-4352, Oct. 1989.

[4] National Instruments PCI-6534, http://www.ni.com

[5] GMH Engineering, http://www.gmheng.com/radar1.html

[6] Microsoft Corporation, http://www.microsoft.com

[7] E.E. Fenimore, T.M. Cannon, "Coded aperture imaging with uniformly redundant arrays," Applied Optics, vol. 17, no. 3, pp. 337-347, Feb., 1978.

[8] C. Brown, "Multiplex Imaging with multiple-pinhole cameras," J. Appl. Phys., Vol. 45, no. 4, pp. 1806-1811, April, 1974.

[9] K. Ziock, "Scintillation imaging: A technique to reduce coding noise in scanned, coded-aperture images," Proc. of SPIE, vol. 5540, pp. 225-234, 2004.

[10] E.E. Fenimore, "Coded aperture imaging: predicted performance of uniformly redundant arrays," Applied Optics, Vol. 17, no. 22, pp. 35623570, Nov., 1978. 